\title{
NEOAJUVANT ENDOCRINE THERAPY IN BREAST CANCER: THE FIRST RESULTS OF 59 PATIENTS
}

Paola Ferreira Freitas, Juliana Costa Souza, Andre Mattar, Jorge Yoshinori Shida, Luiz Henrique Gebrim ${ }^{1}$ Hospital Pérola Byington Hospital, Centro de Referência da Saúde da Mulher - São Paulo (SP), Brazil.

Introduction: Luminal breast cancer cases, those with positive hormone receptors and human epidermal growth factor (HER 2) negative range around $70 \%$ of cases and it is known to be more frequent in older patients. Out of the neoadjuvant therapies, neoadjuvant endocrine therapy (NET) has been gaining more ground due to fewer side effects and tumor downstaging, making it possible for advanced breast cancer tumor patients to undergo breast conservation surgery (BCS). Objectives: To evaluate clinical and pathological response in women that underwent NET with Anastrozole (ANAS) at Pérola Byington Hospital (CRSM), a breast cancer reference center in São Paulo, Brazil. To determine the BCS (breastconserving surgery) rate after NET and the rate of disease progression during treatment. Methods: CRSM patients from 2018 to 2019 that underwent NET for luminal breast cancer with HER 2 negative were identified and data were reviewed. Results: 59 patients were identified, among which only 34 patients underwent breast surgery. All patients were treated with ANAS 1mg/daily. The median age was 63.5 years and average time on treatment was 203.5 days (approximately 6.78 months). Clinical stage at presentation was 3\% Stage I, 6\% Stage IIA, 32\% stage IIB, 43\% stage IIIA and $18 \%$ stage IIIB. After NET we found pathologic complete response (pCR) in $3 \%$ of the cases. and pathological downstage in different stages: Stage I 15\%, Stage IIA 24\%, Stage IIB 26\%, stage IIB 26\%, Stage IIIA 29\% and stage IIIB 3\%. During treatment 6.78\% patients progressed, and all of them received chemotherapy. After NET 71\% were submitted to adjuvant chemotherapy. The change for BCS was $19.35 \%$. During surgical treatment patients $74 \%$ were submitted to axillary dissection and $26 \%$ had sentinel node biopsy (SNB). The average number of SNB removed was 5.33. No patients had to stop the medication due to toxicity. Conclusions: NET is still underutilized, having its optimal period of drug administration range around 8 to 12 months. It has been very important in aiding patients obtain better outcomes allowing for BCS while having fewer side effects when compared to neoadjuvant chemotherapy. 